\title{
BMJ Open Referral patterns, disease progression and impact of the kidney failure risk equation (KFRE) in a Queensland Chronic Kidney Disease Registry (CKD. QLD) cohort: a study protocol
}

\author{
Clyson Mutatiri (D) , ${ }^{1,2}$ Angela Ratsch (D) , ${ }^{3,4}$ Matthew R McGrail, ${ }^{5}$ \\ Sree Venuthurupalli, ${ }^{6,7}$ Srinivas Kondalsamy Chennakesavan ${ }^{7}$
}

To cite: Mutatiri C, Ratsch A, McGrail MR, et al. Referral patterns, disease progression and impact of the kidney failure risk equation (KFRE) in a Queensland Chronic Kidney Disease Registry (CKD.QLD) cohort: a study protocol. BMJ Open 2022;12:e052790. doi:10.1136/ bmjopen-2021-052790

- Prepublication history for this paper is available online To view these files, please visit the journal online (http://dx.doi org/10.1136/bmjopen-2021052790).

Received 27 April 2021 Accepted 24 January 2022

D Check for updates

(c) Author(s) (or their employer(s)) 2022. Re-use permitted under CC BY-NC. No commercial re-use. See rights and permissions. Published by BMJ.

For numbered affiliations see end of article.

\section{Correspondence to}

Dr Clyson Mutatiri;

clyson.mutatirinealth.qld. gov.au

\section{ABSTRACT}

Introduction Chronic kidney disease (CKD) is a rapidly increasing and global phenomenon which carries high morbidity and mortality. Although timely referral from primary care to secondary care confers favourable outcomes, it is not possible for every patient with CKD to be managed at secondary care. With 1 in 10 Australians currently living with markers of CKD against a workforce of about 600 nephrology specialists, a risk stratification strategy is required that will reliably identify individuals whose kidney disease is likely to progress.

Methods and analysis This study will undertake a retrospective secondary analysis of the Chronic Kidney Disease Queensland Registry (CKD.QLD) data of consented adults to examine the referral patterns to specialist nephrology services from primary care providers and map the patient trajectory and outcomes to inform the optimal referral timing for disease mitigation. Patient data over a 5-year period will be examined to determine the impact of the kidney failure risk equation-based risk stratification on the referral patterns, disease progression and patient outcomes. The results will inform considerations of a risk stratification strategy that will ensure adequate predialysis management and add to the discussion of the time interval between referral and initiation of kidney replacement therapy or development of cardiovascular events. Ethics and dissemination This protocol was approved by the Ethics Committee of the Royal Brisbane and Women's Hospital in January 2021 (LNR/2020/QRBW/69707 14/01/2021). The HREC waived the requirement for patient consent as all patients had consented for the use of their data for the purpose of research on recruitment into CKD.QLD Registry. The results will be presented as a component of a PhD study with The University of Queensland. It is anticipated that the results will be presented at health-related conferences (local, national and possibly international) and via publication in peerreviewed academic journals.

\section{INTRODUCTION}

Most individuals with chronic kidney disease (CKD) can be safely managed in primary care. A successful model of primary care

\section{Strengths and limitations of this study}

- The major strength of the current study is the large sample size, derived from one of the largest chronic kidney disease (CKD) surveillance cohorts in the world, with longer follow-up, which provides opportunities to study the journey of patients with CKD under nephrology care and assess several important clinical outcomes and their predictors, including morbidity, mortality, kidney replacement therapy (KRT), hospitalisations and health service consumption.

- Both inter-test and inter-laboratory variability of serum creatinine values may mean that the index estimated glomerular filtration rate at first clinic visit might not necessarily represent baseline kidney function.

- The outcome of initiation of KRT as an endpoint excludes those participants who progressed to end-stage kidney disease (ESKD) but opted to be managed conservatively, which can potentially underestimate the incidence of ESKD in this cohort.

- The participants in the Chronic Kidney Disease in Queensland (CKD.QLD) Registry represent a fraction of individuals with CKD in QLD as the registry data represents $60 \%-70 \%$ of the estimated target population and is limited to individuals with CKD referred to renal services in the public hospital system.

- Given the retrospective and observational nature of the study, it will also be limited by potential confounding, information and selection bias.

should incorporate early detection of the disease through proactive screening of high-risk individuals, together with timely specialist referral of individuals who are at high risk of progressing to end-stage kidney disease (ESKD). ${ }^{1}$ Nevertheless, while the literature suggests that early referral confers favourable outcomes at both individual and system level, ${ }^{2}$ the benefits can be eroded by 
the negative impact of high rates of premature referrals on the healthcare system. ${ }^{3-5}$

Optimal timing for referral of individuals with CKD from primary care to specialist nephrology services has been the subject of debate for several decades and there continues to be a lack of consensus on what constitutes a late (or early) referral. This ambiguity impacts the timing of referral to achieve optimum patient outcomes, which continues to pose an ongoing challenge for patients, policy-makers and service providers.

The increase in the burden of diabetes and hypertension, considered as the two leading drivers of CKD,${ }^{6}$ has led to an increase in individuals diagnosed with CKD in primary care, which in turn has translated into a surge in referrals to specialist nephrology services. ${ }^{7}$ Notably, a significant proportion of these are related to individuals who are at low risk of progressing to ESKD or developing cardiovascular (CV) events. ${ }^{8}$ Compounding the volume rise is the dearth in nephrology specialists in Australia, creating a mismatch between service demand and service providers. A workforce review committee of the Australian and New Zealand Society of Nephrology identified 598 practicing nephrologists in Australia in 2017. ${ }^{9}$ Currently, there are approximately 1.7 million Australians aged $>18$ years with clinical evidence of CKD; specialist referral of all patients with CKD would see an average of $>2800$ patients per nephrologist. ${ }^{10}$ Although the number of Australian nephrology trainees has been expanding at a faster pace in recent years, ${ }^{11}$ the shortage of nephrologists persists outside metropolitan areas in regional, rural and remote areas. ${ }^{9}$ This maldistribution of nephrology workforce is compounded by a higher prevalence of CKD and other chronic conditions in rural and remote areas, where a larger proportion of Aboriginal and Torres Strait Islander peoples reside. ${ }^{12}$

With mounting referrals comes service overload, leading to long wait times and inefficient utilisation of the available nephrologist's time for higher need patients. Moreover, the absense of a precise and easily accessible guide to optimal referral timing escalates the risk that individuals could remain in primary care for too long, resulting in unwanted outcomes for both the affected individual and the healthcare system. To mitigate this situation arising, substantial work has been invested in the development of risk stratification strategies that predict the risk of progression to ESKD as well as CV events, and therefore, the likely need for (more urgent) referral to nephrology services. One such prediction strategy is the kidney failure risk equation (KFRE) which was developed in 2011 to quantify the risk of progression to ESKD. ${ }^{13}$ Despite its validation in North America and Europe, ${ }^{14} 15$ the KFRE has not yet been widely adopted in Australian clinical practice.

In this project, we will conduct a retrospective analysis of an existing Registry, examining participant data and outcomes from the time of the participant's enrolment into the Registry up to and including the end of the fifth year of their follow-up after enrolment. Referral patterns and disease progression will be described, and the KFREbased risk stratification tool will be evaluated against the clinical outcomes.

\section{CKD: epidemiology}

The prevalence of diagnosed CKD has increased worldwide since the adoption of the Kidney Disease Outcomes Quality Initiative classification for CKD, and the implementation of automated reporting of creatinine-based estimated glomerular filtration rate (eGFR) by many laboratories. ${ }^{16}$ Currently, there are nearly 700 million people living with diagnosed CKD across the world, representing a global prevalence of $9.1 \%$ and it is estimated that by 2040, CKD will catapult from its current ranking of twelfth to fifth as the leading cause of mortality around the world. ${ }^{17}$ In Australia, population studies have estimated that every year, at least 16000 Australian adults will be added to the over 1.7 million Australian adults (1 in 10) currently living with biomedical markers of CKD such as a reduced eGFR or protein in their urine .

Given this rise, CKD poses a major challenge to all world healthcare systems and has motivated investments in research and the development of potential strategies to delay or slow progression. ${ }^{18}$ Previous reports by the Australian Institute of Health and Welfare have highlighted that the impact of CKD is not distributed evenly across the population (identifying 'CKD Hot Spots'), with people living in remote areas experiencing substantially higher rates than those living in urban areas.

\section{CKD: identification}

The timely involvement of specialist nephrology services has been shown to improve health outcomes after commencement of kidney replacement therapy (KRT) and can also reduce overall costs of caring for individuals with CKD. ${ }^{19-21}$ In a systematic review of clinical and cost-effectiveness modelling for management of individuals with CKD, the data suggested that early referral strategies may have the potential to offer an efficient use of resources. ${ }^{2}$ Public health campaigns have therefore focused on early detection of CKD in high-risk individuals and also on strategies to reduce the rates of late referrals to nephrology services. ${ }^{22}{ }^{23}$ Nevertheless, determination of the optimal timing of referral to specialist care is complicated by several factors including: the heterogeneity of CKD, the recognised imprecision of estimates of progression based on eGFR trajectories and the nonlinear nature of eGFR decline due to intercurrent events such as acute kidney injury (AKI) or CV events. ${ }^{20} 24$

Knowledge of the trajectory of any disease can form the basis of clinical decision-making by shaping the goals of care and anticipating when interventions might be required. ${ }^{25} 26$ The role of kidney disease trajectories was initially used in clinical practice to predict CKD progression by plotting the reciprocal of serial serum creatinine concentration measurements against time. ${ }^{27}$ Lately, acknowledgement of the substantial heterogeneity of kidney disease trajectories has prompted studies 
to examine the clinical implications of eGFR slopes and their links with subsequent outcomes, and whether past decline in eGFR adds information to the assessment of individuals with kidney disease beyond eGFR at a single time point. ${ }^{28-30} \mathrm{In}$ an international meta-analysis of 22 diverse cohorts consisting of more than a million participants, lower levels of eGFR and a higher decline in eGFR (described as an eGFR slope of $<-5 \mathrm{~mL} / \mathrm{min} / 1.73 \mathrm{~m}^{2} /$ year) were both found to demonstrate a significant and independent association with higher subsequent risk of ESKD in the CKD cohorts. ${ }^{31} \mathrm{~A}$ less than average negative eGFR slope and a positive eGFR slope have previously been found to be associated with increased risks of death and CV events by some investigators, ${ }^{29}{ }^{32-34}$ while a more recent study found no associated risks with eGFR rise or less than average decline in eGFR slopes, suggesting that an improving eGFR might not be associated with adverse outcomes.

\section{CKD: prediction and referral}

Risk prediction models of progression of CKD have been developed to aid treatment decisions and prognostication in clinical practice, hence informing the decision on when to refer from primary care, and on when to refer for access planning in preparation for KRT or transplantation. ${ }^{35}$ With the use of such models, most individuals with lower risk CKD (eg, stage G3) can potentially be treated solely by their primary care provider, whereas those at high risk of progression to ESKD should be referred for specialised care by nephrology services .

Many different risk prediction equations have been developed and a few of them, including the KFRE, have been validated in different CKD cohorts. ${ }^{136}$ The accuracy of these equations for predicting risk of kidney failure was evaluated by Tangri et al in a meta-analysis involving 31 cohorts, including 721357 participants with CKD stages G3-G5 in more than 30 countries spanning 4 continents. From the meta-analysis, 3 ESKD prediction equations were derived and assessed, based on 4, 6 or 8 variables and the performance of the 4-variable KFRE was found to be similar to that of the other 2 equations. ${ }^{37}$ In another study which undertook external validation of 11 existing models of kidney failure, the 4 and 8 -variable 2-year KFREs were found to be most suitable for short-term prediction of risk of kidney failure. However, for prediction of kidney failure over a longer time frame, the 5-year KFRE overestimated the actual risk of KRT by $10 \%-18 \%$ due to the competing risk of death. ${ }^{38}$ Furthermore, the application of the KFRE was explored more recently by Naranjo et al. Using electronic medical records to estimate the risk of kidney failure, the 4-variable KFRE (with albuminuria) resulted in consistent improvement in risk discrimination when compared with the 3-variable KFRE (without albuminuria), even when albumin-creatinine ratio (ACR) was imputed from protein-creatinine ratio (PCR) or urine dipstick protein measurements. ${ }^{39}$ These findings were consistent with previous studies that have concluded that ascertainment of albuminuria is central to ESKD prognosis. ${ }^{13} 37$

The 4-variable KFRE can be easily implemented in electronic medical records and laboratory information systems and has therefore been recommended as the model for implementation into clinical practice. ${ }^{37}$ In its simplest and most common application, the 4-variable KFRE requires the input of age, gender, Chronic Kidney Disease Epidemiology Collaboration (CKDEPI) creatinine-based eGFR and urine $\mathrm{ACR}^{40}$ In addition to its use for predicting referral from primary care to nephrology services, the KFRE may also be used to improve timing of referral for permanent vascular access creation and kidney transplantation. ${ }^{41}$

An examination of data collected at state level and analysed against the KFRE could assist in identifying or mapping the distribution of state-wide CKD and assessing the usefulness of the tool to support health service planning at the state and local level.

\section{Data source-CKD.QLD Registry}

The Chronic Kidney Disease in Queensland (CKD.QLD) Registry is a collaborative of most public sector nephrology practices in Queensland, Australia. The main objective of the Registry is to profile all consenting participants with CKD, laying a foundation for CKD surveillance, practice improvement and research. The CKD.QLD data collection methods have been described by Venuthurapalli $e t$ $a l$, briefly however, the Registry was designed to use a data linkage framework which centralises data captured by multiple mechanisms to an individual participant via a unique identifier. ${ }^{42}$ Patients already on kidney replacement therapy and those with AKI are excluded unless they subsequently developed and met the diagnostic criteria for CKD. After commencing in May 2011, recruitment of new participants to the Registry was discontinued in May 2019 on the advice of the CKD.QLD surveillance stream due to funding constraints. However, participating sites who wished to continue enrolling new participants could do so if they had a special reason. Sites that ceased enrolment could still take up recruitment again, with governance and ethics approvals refreshed, if they later chose to do so, but analyses would be their own responsibilities. Some sites chose to continue, as they found the registry functions useful as an audit tool.

\section{METHODS AND DESIGN}

This is a retrospective study which will undertake a secondary analysis of CKD.QLD Registry data. The aims and hypotheses of this study are:

\section{Aims}

- To describe the referral patterns of participants in the CKD.QLD database with regard to the timing and appropriateness of referral and the associated impact on outcomes. 


\section{Box 1 Indications for referral to a nephrologist}

- Estimated glomerular filtration rate (eGFR) $<30 \mathrm{~mL} / \mathrm{min} / 1.73 \mathrm{~m}^{2}$ (stage G4 or G5 chronic kidney disease (CKD) of any cause)

- Persistent significant albuminuria (urine albumin-creatinine ratio $\geq 30 \mathrm{mg} / \mathrm{mmol}$ )

- A sustained decrease in eGFR of $25 \%$ or more within 12 months OR a sustained decrease in eGFR of $15 \mathrm{~mL} / \mathrm{min} / 1.73 \mathrm{~m}^{2}$ per year

- CKD with hypertension that is hard to get to target despite at least three antihypertensive agents

- To study the progression of CKD in a subpopulation of participants followed up in specialist nephrology clinics. This includes comparing the association of prereferral eGFR slope with subsequent adverse outcomes between early referrals and late referrals and examining the consistency of these associations across subgroups.

- To evaluate the application of the KFRE and its impact on referral patterns.

\section{Hypotheses}

- For participants with CKD, timely referral to specialist care will be associated with slowing down of progression to ESKD, improvement in CV outcomes and efficient utilisation of resources.

- For participants under the care of nephrology services, past eGFR slope and albuminuria category are associated with the rate of progression of CKD and subsequent clinical outcomes.

- For participants with CKD, the KFRE will significantly increase identification of those who are at risk of progressing to ESKD, and who would benefit from timely referral to specialist nephrology services.

\section{Sampling framework and study participants}

Overall, the Registry includes approximately 7600 participants $\geq 18$ years who were enrolled between 1 January 2011 and 31 December 2018. The participants were drawn from patients attending public kidney clinics in QLD Health facilities; thus, the Registry contains a mix of prevalent and incident patients. All such participants will contribute to completing aim 1, while aim 2 limits participants to those who were enrolled in the CKD.QLD Registry between 1 June 2011 and 30 June 2013 and followed up for at least 5years, until 31 December 2018. Approval to access the CKD.QLD data is under the participant's original consent to share their data for research, and access to hospital record(s) has been granted under a waiver of consent by the Royal Brisbane and Women's Hospital Human Research Ethics Committee (LNR/2020/QRBW/69707 14/01/2021).

\section{Research outcomes}

There are a number of outcomes of interest to this study's aims and these are defined below:

\section{Referral patterns}

- Prevalence of late referrals by eGFR.
- Describe outcomes associated with late referral.

- Proportion of participants who progressed to KRT at 3, 6 and 12 months.

- Proportion of participants who commenced KRT with a temporary vascular access.

- Incident KRT modality (haemodialysis versus peritoneal dialysis versus pre-emptive transplantation).

- Characteristics of participants who are referred late.

Progression of CKD under specialist nephrology services

- Association of past eGFR slopes versus eGFR at referral, albuminuria stage, comorbidities and cause of CKD with subsequent outcomes (ESKD, non-CV death and major adverse cardiovascular events (MACE).

- Five-year cumulative incidence of KRT, MACE, death without ESKD and KRT-free survival by past eGFR slope, eGFR and albuminuria stage at study entry.

- Correlation of eGFR slope with the time interval between referral and initiation of KRT.

- Outcomes at 3,6 and 12 months after first visit to nephrology service (MACE, KRT, hospitalisation).

\section{Impact of the KFRE on referral patterns}

- The proportion of participants in the database who require redesignation of their referrals to the nephrologist using the KFRE.

- The number of participants in the database who met the Kidney Health Australia (KHA)'s recommendations for nephrology referral.

- The proportion of participants who required referral for access creation and the timing of referral as predicted by the KFRE.

- The proportion of participants who progressed to ESKD as predicted by the KFRE and the time it took to progress to ESKD from time of referral.

- The proportion of participants in the database who had a low risk of progression at baseline and could therefore have been managed safely by their primary care providers.

- The effect of the KFRE stratification on number of referrals and wait times.

\section{Definitions related to referral}

\section{Appropriateness of referral}

The appropriateness of referrals will be determined according to the recommendations in KHA's The Chronic Kidney Disease Management in Primary Care handbook, ${ }^{43}$ which are also in tandem with the Caring for Australian and New Zealanders with Kidney Impairment (CARI) guidelines ${ }^{44}$ (box 1). Referrals will be deemed as appropriate if any of the indications for referral are met.

\section{Definition of late referral}

Kidney Disease: Improving Global Outcomes defines late referral as referral to specialist services less than 1 year before start of KRT. ${ }^{45}$ However, many previous studies have applied a cut-off of less than 3-4 months, with others going for less than 6 months. In our analysis, we plan to apply the 3 months cut-off as this is the definition that is 
Table 1 Demographic and clinical characteristics of the study participants

\begin{tabular}{|c|c|c|c|}
\hline Variable & Operational definition & $\begin{array}{l}\text { Scale of } \\
\text { measurement }\end{array}$ & Collection interval \\
\hline Gender & Will be taken as recorded in database & $\mathrm{M}$ or $\mathrm{F}$ or other & Baseline \\
\hline $\begin{array}{l}\text { Indigenous } \\
\text { status }\end{array}$ & Indigenous versus non-Indigenous & $\mathrm{Y}$ or $\mathrm{N}$ & $\begin{array}{l}\text { Baseline, at } 3 \text { and } 6 \\
\text { months and } 12 \text { monthly }\end{array}$ \\
\hline $\begin{array}{l}\text { Area of } \\
\text { residence }\end{array}$ & Area of residence by postcode & Rural versus urban & $\begin{array}{l}\text { Baseline, at } 3 \text { and } 6 \\
\text { months and } 12 \text { monthly }\end{array}$ \\
\hline Wait times & Time from time of referral to first visit to the kidney clinic & Number of months & Baseline \\
\hline T2DM & $\begin{array}{l}\text { Clinical label of T2DM or no requirement of insulin within } 1 \text { year } \\
\text { of diagnosis of DM }\end{array}$ & Y or $\mathrm{N}$ & $\begin{array}{l}\text { Baseline, at } 3 \text { and } 6 \\
\text { months and } 12 \text { monthly }\end{array}$ \\
\hline Obesity & $\mathrm{BMI} \geq 30$ & $\mathrm{Y}$ or $\mathrm{N}$ & Baseline \\
\hline Dyslipidaemia & $\begin{array}{l}\text { Dyslipidaemia will be defined as a LDL-C of } \geq 2.586 \text { without } \\
\text { further risk factors and } \geq 1.81 \text { in patients with CVD or CKD or } \\
\text { receipt of lipid lowering drug treatment }\end{array}$ & $\mathrm{Y}$ or $\mathrm{N}$ & Baseline \\
\hline $\mathrm{CHD}$ & $\begin{array}{l}\text { History of acute myocardial infarction or history of coronary } \\
\text { revascularisation }\end{array}$ & $\mathrm{Y}$ or $\mathrm{N}$ & $\begin{array}{l}\text { Baseline, at } 3 \text { and } 6 \\
\text { months and } 12 \text { monthly }\end{array}$ \\
\hline Heart failure & $\begin{array}{l}\text { The diagnosis of heart failure will be obtained from participant } \\
\text { admission records }\end{array}$ & $\mathrm{Y}$ or $\mathrm{N}$ & $\begin{array}{l}\text { Baseline, at } 3 \text { and } 6 \\
\text { months and } 12 \text { monthly }\end{array}$ \\
\hline Smoking & Smoking status & $\begin{array}{l}\text { Former or current or } \\
\text { never }\end{array}$ & Baseline \\
\hline $\begin{array}{l}\text { Pulmonary } \\
\text { disease }\end{array}$ & Chronic obstructive pulmonary disease or emphysema & $\mathrm{Y}$ or $\mathrm{N}$ & $\begin{array}{l}\text { Baseline, at } 3 \text { and } 6 \\
\text { months and } 12 \text { monthly }\end{array}$ \\
\hline Other diseases & As documented in participant record. & $\mathrm{Y}$ or $\mathrm{N}$ & $\begin{array}{l}\text { Baseline, at } 3 \text { and } 6 \\
\text { months and } 12 \text { monthly }\end{array}$ \\
\hline
\end{tabular}

BMI, body mass index; BP, blood pressure; CHD, coronary heart disease; CKD, chronic kidney disease; CVA, cerebrovascular accident; CVD, cardiovascular disease; DBP, diastolic blood pressure; DM, diabetes mellitus; LDL-C, low-density lipoprotein cholesterol; PVD, peripheral vascular disease; SBP, systolic blood pressure; SEIFA, Socio-Economic Indexes for Areas; SES, socioeconomic status; T1DM, type 1 diabetes mellitus; T2DM, type 2 diabetes mellitus; TIA, transient ischaemic attack.

used by KHA in their latest edition of their CKD management in primary care handbook. ${ }^{43}$

Kidney failure risk equation

The published KFRE 4-variable non-North American equation will be used at the initial visit to discriminate participants who would develop kidney failure within 5 years from those who would not. The observed kidney failure rate (on the basis of CKD.QLD follow-up data) will be the reference. The variables accessible in the CKD.
QLD database will allow for integration into the KFRE to calculate the proportion of patients who would fulfil criteria for referral to the nephrologist based on the calculated risk. eGFR (calculated from serum creatinine using the CKD-EPI equation), urine ACR (where available), gender and age will be retrieved from the Registry to enable the calculation of each patient's risk threshold for progression to ESKD. Where only results of urine PCR or urine dipsticks are available, the equations developed 
Table 2 Primary cause of chronic kidney disease at referral

\begin{tabular}{ll}
\hline Primary kidney disease & Duration \\
\hline DKD & Date of first diagnosis or if not available, duration prior to entering the CKD Registry \\
\hline Glomerulonephritis & Date of first diagnosis or if not available, duration prior to entering the CKD Registry \\
\hline Obstructive uropathy & Date of first diagnosis or if not available, duration prior to entering the CKD Registry \\
\hline Hypertensive kidney disease & Date of first diagnosis or if not available, duration prior to entering the CKD Registry \\
\hline ADPKD & Date of first diagnosis or if not available, duration prior to entering the CKD Registry \\
\hline ANCA vasculitis & Date of first diagnosis or if not available, duration prior to entering the CKD Registry \\
\hline Lupus nephritis & Date of first diagnosis or if not available, duration prior to entering the CKD Registry \\
\hline Renovascular disease & Date of first diagnosis or if not available, duration prior to entering the CKD Registry \\
\hline Reflux nephropathy & Date of first diagnosis or if not available, duration prior to entering the CKD Registry \\
\hline Other & Date of first diagnosis or if not available, duration prior to entering the CKD Registry \\
\hline Unknown & Date of first diagnosis or if not available, duration prior to entering the CKD Registry
\end{tabular}

The primary cause of chronic kidney disease (CKD) will be based on the entry by the treating nephrologist.

ADPKD, autosomal dominant polycystic kidney disease; ANCA, antineutrophil cytoplasmic antibodies; DKD, diabetic kidney disease.

by Sumida et al in 2020 will be employed to calculate the predicted ACR. ${ }^{46}$ Where no ACR, PCR or urine dipsticks are available at the initial visit, we intend to use the earliest interval where the first urine protein examination would have been performed within the first 6 months of the initial visit. We anticipate that most patients seen in the nephrology clinics will be brought back for review within 3-6 months and that most of them will have urine examination for proteinuria/albuminuria ordered by the nephrologist. The date of the proteinuria/albuminuria measurement will automatically become the date for estimating baseline risk using the KFRE and for beginning the follow-up period.

The participants will then be stratified as either high risk or low risk according to the calculated 2-year and 5-year risk of progressing to ESKD. Patients whose 5-year risk for kidney failure is less than $3 \%$ and are without any structural abnormalities such as a diagnosis of polycystic kidney disease would be deemed low risk and hence could be safely managed in primary care, whereas all those with a 5-year risk of $>3 \%$ would be classified as high risk and therefore would be considered for a nephrology referral. A KFRE threshold of $>10 \%$ in 2 years would require referral to multidisciplinary team programmes, whereas a 2-year threshold of 20\%-40\% would trigger referral for planning a transplant or fistula. ${ }^{5478}$

\section{Procedure}

The CKD.QLD data custodian will provide the researchers with the CKD.QLD identification number of the eligible population and will provide access to the Registry data set. Each participant's CKD.QLD identification number will be matched with QLD hospital record numbers. The variables to be extracted from CKD.QLD and hospitalisation records are listed in tables 1-6 and will be entered

Table 3 Pathology results to be collected

\begin{tabular}{|c|c|c|}
\hline Laboratory parameter & Units of measurement & Collection interval \\
\hline Urine albumin to creatinine ratio & $\mathrm{mg} / \mathrm{mmol}$ or $\mathrm{g} / \mathrm{mol}$ & Baseline, at 3 and 6 months and 12 monthly \\
\hline Urine protein to creatinine ratio & $\mathrm{mg} / \mathrm{mmol}$ or $\mathrm{g} / \mathrm{mol}$ & Baseline, at 3 and 6 months and 12 monthly \\
\hline Urine dipstick & Negative or positive for protein & Baseline, at 3 and 6 months and 12 monthly \\
\hline Serum creatinine & micromol/L & Baseline, at 3 and 6 months and 12 monthly \\
\hline Serum albumin & $g / L$ & Baseline, at 3 and 6 months and 12 monthly \\
\hline $\mathrm{Hb}$ & g/L & Baseline, at 3 and 6 months and 12 monthly \\
\hline Serum calcium & $\mathrm{mmol} / \mathrm{L}$ & Baseline, at 3 and 6 months and 12 monthly \\
\hline Serum phosphate & $\mathrm{mmol} / \mathrm{L}$ & Baseline, at 3 and 6 months and 12 monthly \\
\hline Serum PTH & $\mathrm{pmol} / \mathrm{L}$ & Baseline, at 3 and 6 months and 12 monthly \\
\hline
\end{tabular}

eGFR, estimated glomerular filtration rate; $\mathrm{Hb}$, haemoglobin; HbA1C, glycated haemoglobin; PTH, parathyroid hormone. 
Table 4 Parameters of prereferral management

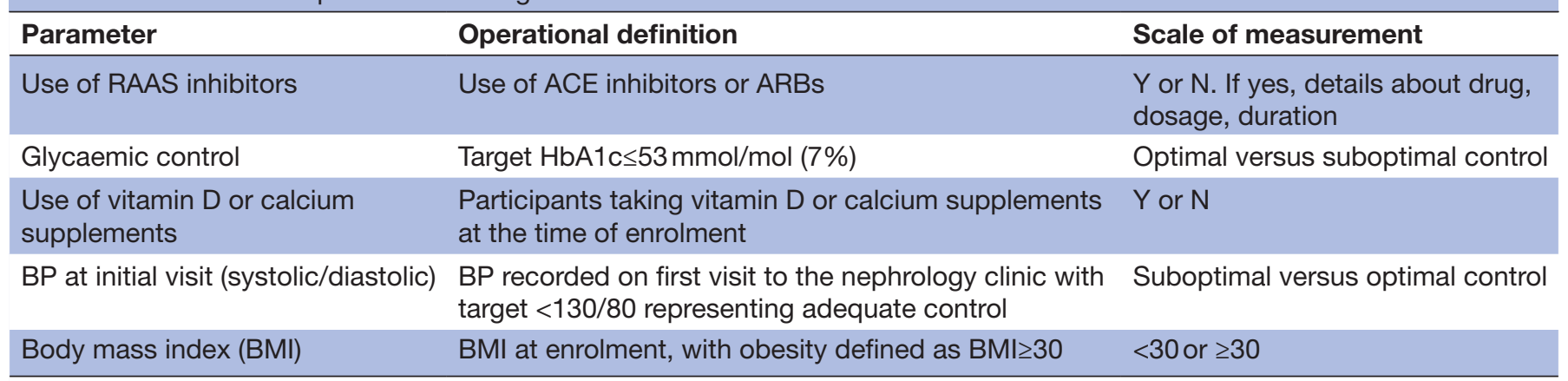

ARB, angiotensin receptor blockers; BP, blood pressure; HbA1c, glycated haemoglobin; RAAS, renin angiotensin aldosterone system.

onto Excel spreadsheets prior to upload in STATA V.16.0 (StataCorp LP. Stata Statistical Software).

\section{Data analysis}

This is an observational study and a range of statistical methods will be used to measure the association between data elements and outcomes. First, descriptive statistics and basic inferential statistics will be used to present participant characteristics and explore basic patterns of the data (eg, describing referral patterns). If the variables are continuous and normally distributed, means and t-tests will be used. For non-normally distributed (skewed) continuous data, medians and equivalent non-parametric tests will be used. For categorical data, proportions and $\chi^{2}$ tests will be used and reported.

Second, where appropriate, multivariate analysis methods will be used to account for measurable covariates, thus better capturing the true effect size. Multiple regression models (linear, multinomial, logistic and Cox proportional hazards or competing risks, where appropriate) will be used to explore the association between participant characteristics and clinical outcomes adjusting for potential confounders based on previous literature and available measures as outlined in table $1 .{ }^{49}{ }^{50}$ Variables will be fitted as covariates in the regression models and variables with a $\mathrm{p}<0.2$ will be accepted for covariate interaction inclusion in the regression model.

The variables broadly categorised as follows will be investigated as any of predictors, covariates or outcomes, as appropriate to the analyses:

- Demographic variables (age, residential address, gender, ethnicity, socioeconomic status).

- Lifestyle factors (alcohol and tobacco use).

Table 5 Participant outcomes under nephrological care

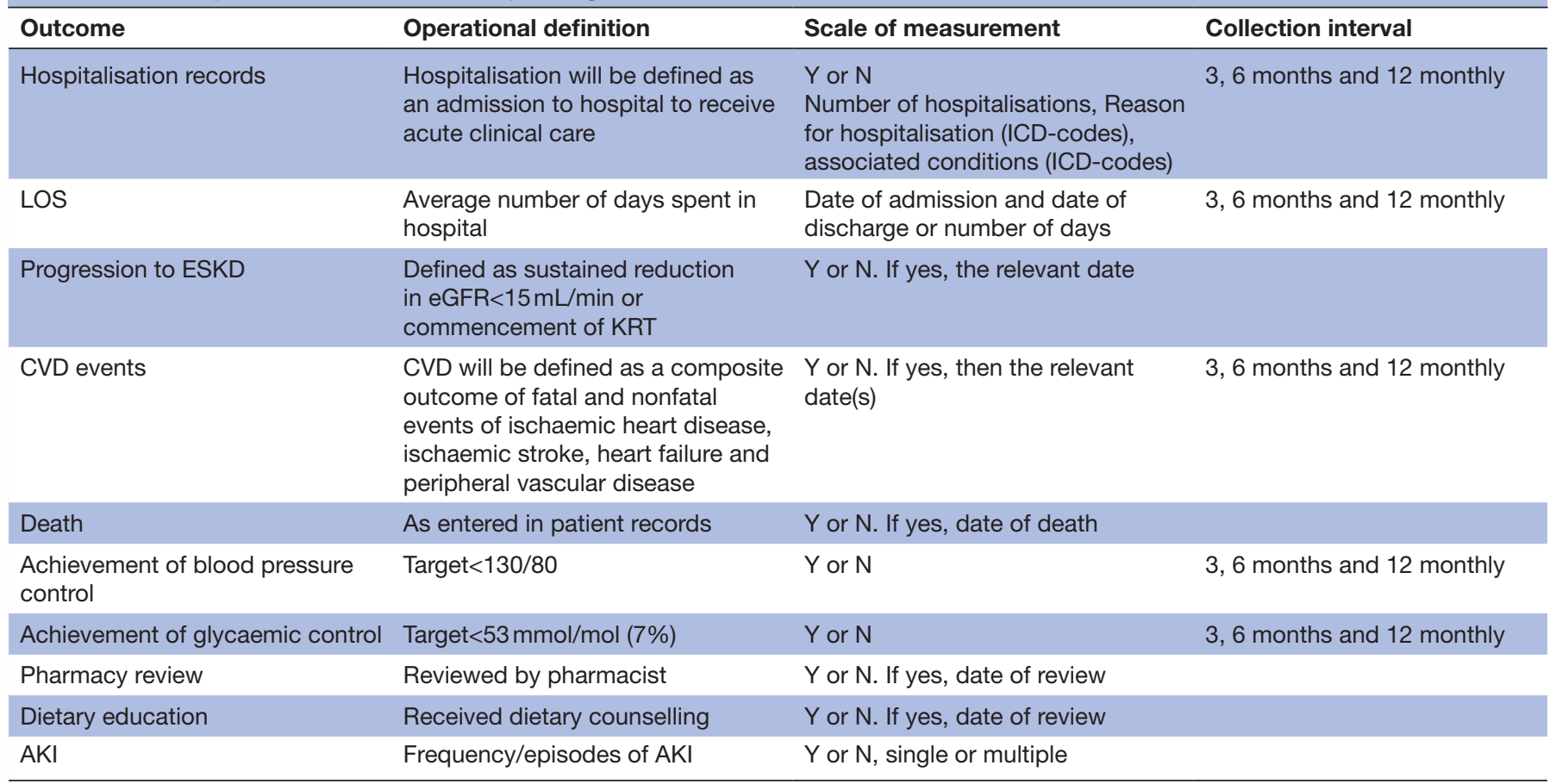

AKI, acute kidney injury; CVD, cardiovascular disease; eGFR, estimated glomerular filtration rate; ESKD, end-stage kidney disease; ICD, International Classification of Diseases; KRT, kidney replacement therapy; LOS, length of stay. 
Table 6 Pharmacological intervention after referral

\begin{tabular}{|c|c|c|}
\hline Medication initiated & Operational definition & Scale of measurement \\
\hline ESA therapy & $\begin{array}{l}\text { Initiation of ESA in participants whose } \mathrm{Hb} \text { is } \\
<100 \mathrm{~g} / \mathrm{L}\end{array}$ & $\begin{array}{l}\text { Y or } \mathrm{N} \text {. If yes, date of initiation or } \\
\text { titration if available }\end{array}$ \\
\hline Other antihypertensive medication & Initiation of other antihypertensive medication & $\mathrm{Y}$ or $\mathrm{N}$. If yes, date of initiation \\
\hline Calcium supplements & Calcium supplements & $\mathrm{Y}$ or $\mathrm{N}$. If yes, date of initiation \\
\hline Loop diuretics & Loop diuretics & $\mathrm{Y}$ or $\mathrm{N}$. If yes, date of initiation \\
\hline
\end{tabular}

ACE-I, ACE inhibitors; ARB, angiotensin receptor blockers; ESA, erythropoiesis stimulating agent; Hb, haemoglobin.

- Past and current medical history (cardiac, hypertension, diabetes and type and level of kidney disease, pulmonary disease, obesity).

- Kidney disease factors (urine albumin/creatinine ratio, urine protein/creatinine ratio, 24 hours urine protein excretion, serum creatinine, eGFR, glycated haemoglobin, serum phosphate, parathyroid hormone (PTH), haemoglobin, serum albumin).

- Kidney disease outcome variables (transplant, KRT, dialysis modality, death).

- Prereferral parameters (renin angiotensin aldosterone system inhibitors, lipid lowering drugs, vitamin D or calcium supplements, systolic blood pressure (BP), diastolic BP, BMI, PTH, serum albumin, eGFR, serum calcium, serum phosphate, urine ACR, urine PCR, 24 hours protein excretion, urine dipstick, haemoglobin).

Results will be reported with the level of significance at alpha 0.05 and accompanied by $95 \%$ CI. Missing data and outliers will be reported but will be excluded from analysis. Outliers will be identified by the use of boxplots in STATA. These outliers will be confirmed based on the established procedures from Hoaglin and Iglewicz. ${ }^{51}$

\section{Benefits and policy implications}

Determination of the optimal risk stratification strategy which will accurately predict risk of progression to ESKD or development of CV events will inform the optimal timing of referral to specialist care. This has several policy implications in the overall care of the patient with CKD including:

1. Spurring the need to review referral strategies, guidelines, and QLD health service delivery recommendations to improve the health outcomes of CKD and maximise efficiency of its management within the healthcare system.

2. Balancing of quality of care and cost by improving appropriateness of referrals and efficient integration of primary and specialist services.

3. Enabling the coordination of primary care and secondary care providers to optimise value by providing clinically indicated services at the appropriate time and reducing rates of referrals of low risk patients who could be safely managed in primary care.
4. Reserving scarce specialist services for individuals at high risk of progressing to ESKD or developing CV events, where timely intervention is likely to improve outcomes.

5. Helping to inform the need for education of healthcare providers and implementation of targeted care initiatives, to ensure that individuals at high risk of progressing to ESKD are captured earlier and given an opportunity to benefit from a more specialised care environment.

Ultimately, the study findings are intended to provide CKD healthcare providers with a robust decision-making tool. This will enable targeted care initiatives to ensure that individuals at high risk of progressing to ESKD are identified early and given an opportunity to benefit from specialised nephrology care.

\section{Patient and public involvement}

No patients were involved in the development of the research questions or the design and development of the study protocol. However, the conception of the study protocol, the scope of the research questions and outcome measures were informed by identified gaps in the current specialist referral process of individuals with CKD, and the uncertainty of the optimal timing of referral that will enable optimal specialist intervention, all of which were inspired by interaction with patients in the nephrology clinics. Results will be disseminated to patients and the public through social media and through their primary care physicians.

\section{Ethics and dissemination}

\section{Ethics}

The CKD.QLD Registry and the hospital record(s) are being examined by the researchers retrospective to the participants' details being entered into CKD.QLD and their hospital admissions. The CKD.QLD data and the hospital record(s) are identifiable data, critical to the data linkage of this research. On enrolment into CKD. QLD, the informed consent for enrolment for the CKD. QLD Registry included permission to access and link all relevant clinical material on the participants, including medical history, pathology reports and hospital admissions collected prior to enrolment in the registry for future CKD research. The Participant Information and Consent Form includes the following statement: 'The 
(CKD.QLD Registry) information is used for improvement of the quality of care for people with kidney disease, to study kidney disease and plan health services' and 'The information produced from the database may be used for future research in CKD. However, any research proposal based on the information collected from you will require additional approval from Ethics committees belonging to Queensland Health'.

\section{Dissemination}

The results will be presented as a component of a $\mathrm{PhD}$ study with The University of Queensland. It is also anticipated that the results will be presented at health-related conferences (local, national and possibly international) and via publication in peer-reviewed academic journals.

\section{Author affiliations}

${ }^{1}$ Renal Medicine, Wide Bay Hospital and Health Service, Bundaberg, Queensland, Australia

${ }^{2}$ Rural Clinical School, Faculty of Medicine, The University of Queensland, Bundaberg, Queensland, Australia

${ }^{3}$ Research Services, Wide Bay Hospital and Health Service, Hervey Bay, Queensland, Australia

${ }^{4}$ Rural Clinical School, Faculty of Medicine, The University of Queensland, Hervey

Bay, Queensland, Australia

${ }^{5}$ Rural Clinical School, Faculty of Medicine, The University of Queensland, Rockhampton, Queensland, Australia

${ }^{6}$ Kidney Service, Department of Medicine, West Moreton Hospital and Health Service, Ipswich, Queensland, Australia

${ }^{7}$ Rural Clinical School, Faculty of Medicine, The University of Queensland, Toowoomba, Queensland, Australia

\section{Twitter Clyson Mutatiri @cj19632}

Acknowledgements We would like to thank the CKD.QLD Registry for granting us the approval of access to CKD.QLD Registry Patient Data and support for the progression of this research. We would also like to acknowledge the 'in-kind' support provided by Wide Bay Hospital and Health Service to the researchers and for relevant resources including computers, telephone, and office space for the duration of this project. This study was partly supported by the Australian Government Department of Health under the Rural Health Multidisciplinary Training Programme.

Contributors Conceptualisation: CM, SKC, SV and AR; methodology: all authors; writing —original draft preparation: CM, SKC, AR; writing—review and editing: AR, SKC, MRMG, SV. All authors have read and agreed to the published version of the manuscript.

Funding The APC was partially funded by Otsuka Australia (Award/Grant number is not applicable for this funding).

Competing interests None declared.

Patient and public involvement Patients and/or the public were not involved in the design, or conduct, or reporting, or dissemination plans of this research.

Patient consent for publication Not applicable.

Provenance and peer review Not commissioned; externally peer reviewed.

Open access This is an open access article distributed in accordance with the Creative Commons Attribution Non Commercial (CC BY-NC 4.0) license, which permits others to distribute, remix, adapt, build upon this work noncommercially, and license their derivative works on different terms, provided the original work is properly cited, appropriate credit is given, any changes made indicated, and the use is non-commercial. See: http://creativecommons.org/ licenses/by-nc/4.0/.

\section{ORCID iDs}

Clyson Mutatiri http://orcid.org/0000-0003-3033-2842

Angela Ratsch http://orcid.org/0000-0003-0806-6293
REFERENCES

1 Kamath CC, Dobler CC, Lampman MA, et al. Implementation strategies for interventions to improve the management of chronic kidney disease (CKD) by primary care clinicians: protocol for a systematic review. BMJ Open 2019;9:e027206.

2 Black C, Sharma P, Scotland G, et al. Early referral strategies for management of people with markers of renal disease: a systematic review of the evidence of clinical effectiveness, cost-effectiveness and economic analysis. Health Technol Assess 2010;14:1-184.

3 Moynihan R, Glassock R, Doust J. Chronic kidney disease controversy: how expanding definitions are unnecessarily labelling many people as diseased. BMJ 2013;347:f4298.

4 Foote C, Clayton PA, Johnson DW, et al. Impact of estimated GFR reporting on late referral rates and practice patterns for end-stage kidney disease patients: a multilevel logistic regression analysis using the Australia and New Zealand dialysis and transplant registry (ANZDATA). Am J Kidney Dis 2014;64:359-66.

5 Hingwala J, Wojciechowski P, Hiebert B, et al. Risk-based triage for nephrology referrals using the kidney failure risk equation. Can $J$ Kidney Health Dis 2017;4:205435811772278.

6 Xie Y, Bowe B, Mokdad AH, et al. Analysis of the Global Burden of Disease study highlights the global, regional, and national trends of chronic kidney disease epidemiology from 1990 to 2016. Kidney Int 2018;94:567-81.

7 Martínez-Castelao A, Soler MJ, Górriz Teruel JL, et al. Optimizing the timing of nephrology referral for patients with diabetic kidney disease. Clin Kidney J 2021;14:5-8.

8 Go AS, Yang J, Tan TC, et al. Contemporary rates and predictors of fast progression of chronic kidney disease in adults with and without diabetes mellitus. BMC Nephrol 2018;19:1-13.

9 Australian and New Zealand Society of nephrology. Report of the workforce review Committee of the Australian and New Zealand Society of nephrology, 2021. Available: https://www.nephrology.edu. au/workforce-report.asp [Accessed 08 Mar 2021].

10 Australian Institute of Health and Welfare. Chronic kidney disease. Available: https://www.aihw.gov.au/reports/chronic-kidney-disease/ chronic-kidney-disease [Accessed 07 Mar 2021].

11 Amos L, Toussaint ND, Phoon RK, et al. Increase in nephrology advanced trainee numbers in Australia and associated reduction in clinical exposure over the past decade. Intern Med $J$ 2013;43:287-93.

12 Wright J, Glenister K, Thwaites R, et al. The importance of adequate referrals for chronic kidney disease management in a regional Australian area of nephrologist workforce shortage. Aust J Gen Pract 2018;47:58-62.

13 Tangri N, Stevens LA, Griffith J. A predictive model for progression of chronic kidney disease to kidney failure. JAMA 2011;305:1553-9.

14 Major RW, Shepherd D, Medcalf JF, et al. The kidney failure risk equation for prediction of end stage renal disease in UK primary care: an external validation and clinical impact projection cohort study. PLoS Med 2019;16:e1002955.

15 Whitlock RH, Chartier M, Komenda P, et al. Validation of the kidney failure risk equation in Manitoba. Can J Kidney Health Dis 2017;4:205435811770537-9.

16 Hemmelgarn BR, Zhang J, Manns BJ, et al. Nephrology visits and health care resource use before and after reporting estimated glomerular filtration rate. JAMA 2010;303:1151-8.

17 Foreman KJ, Marquez N, Dolgert A, et al. Forecasting life expectancy, years of life lost, and all-cause and cause-specific mortality for 250 causes of death: reference and alternative scenarios for 2016-40 for 195 countries and territories. The Lancet 2018;392:2052-90.

18 Ojo A. Addressing the global burden of chronic kidney disease through clinical and translational research. Trans Am Clin Climatol Assoc 2014;125:229-46.

19 Wouters OJ, O'Donoghue DJ, Ritchie J, et al. Early chronic kidney disease: diagnosis, management and models of care. Nat Rev Nephrol 2015;11:491-502.

20 Stroupe KT, Fischer MJ, Kaufman JS, et al. Predialysis nephrology care and costs in elderly patients initiating dialysis. Med Care 2011;49:248-56.

21 Levin A, Stevens PE, Bilous RW. Kidney disease: improving global outcomes (KDIGO) CKD work group. KDIGO 2012 clinical practice guideline for the evaluation and management of chronic kidney disease. Kidney Int 2013;3:1-150.

22 Li PK-T, Garcia-Garcia G, Lui S-F, et al. Kidney Health for Everyone Everywhere - From Prevention to Detection and Equitable Access to Care. Blood Purif 2021;50:1-8.

23 Ryz K, Tangri N, Verrelli M, et al. A before and after cross-sectional analysis of a public health campaign to increase kidney health awareness in a Canadian Province. BMC Res Notes 2015;8:1-6. 
24 Kaushal A, Naimark D, Tangri N. Use of the kidney failure risk equation to reduce uncertainty in predicting time to ESRD. Am J Kidney Dis 2015;65:369-71.

25 Gill TM, Gahbauer EA, Han L, et al. Trajectories of disability in the last year of life. N Engl J Med 2010;362:1173-80.

26 Lunney JR, Lynn J, Foley DJ, et al. Patterns of functional decline at the end of life. JAMA 2003;289:2387-92.

27 Mitch WE, Walser M, Buffington GA, et al. A simple method of estimating progression of chronic renal failure. Lancet 1976;2:1326-8.

28 Inker LA, Heerspink HJL, Tighiouart $\mathrm{H}$, et al. Gfr slope as a surrogate end point for kidney disease progression in clinical trials: a metaanalysis of treatment effects of randomized controlled trials. J Am Soc Nephrol 2019;30:1735-45.

29 Naimark DMJ, Grams ME, Matsushita K, et al. Past decline versus current eGFR and subsequent mortality risk. J Am Soc Nephrol 2016;27:2456-66.

30 Levey AS, Inker LA, Matsushita K, et al. GFR decline as an end point for clinical trials in CKD: a scientific workshop sponsored by the National kidney Foundation and the US food and drug administration. Am J Kidney Dis 2014;64:821-35.

31 Kovesdy CP, Coresh J, Ballew SH, et al. Past decline versus current eGFR and subsequent ESRD risk. J Am Soc Nephrol 2016;27:2447-55.

32 Perkins RM, Bucaloiu ID, Kirchner HL, et al. GFR decline and mortality risk among patients with chronic kidney disease. Clin J Am Soc Nephrol 2011;6:1879-86.

33 Al-Aly Z, Zeringue A, Fu J, et al. Rate of kidney function decline associates with mortality. J Am Soc Nephrol 2010;21:1961-9.

34 Coresh J, Turin TC, Matsushita K, et al. Decline in estimated glomerular filtration rate and subsequent risk of end-stage renal disease and mortality. JAMA 2014;311:2518-31.

35 Lerner B, Desrochers S, Tangri N. Risk prediction models in CKD. In Proceedings of Semin Nephrol, 2021: 144-50.

36 Tangri N, Kitsios GD, Inker LA, et al. Risk prediction models for patients with chronic kidney disease: a systematic review. Ann Intern Med 2013;158:596-603.

37 Tangri N, Grams ME, Levey AS, et al. Multinational assessment of accuracy of equations for predicting risk of kidney failure: a metaanalysis. JAMA 2016;315:164-74.

38 Ramspek CL, Evans M, Wanner C, et al. Kidney failure prediction models: a comprehensive external validation study in patients with advanced CKD. J Am Soc Nephrol 2021;32:1174-86.
39 Naranjo FS, Sang Y, Ballew SH, et al. Estimating kidney failure risk using electronic medical records. Kidney360 2021;2:415-24.

40 McCudden C, Akbari A, White CA, et al. Individual patient variability with the application of the kidney failure risk equation in advanced chronic kidney disease. PLoS One 2018;13:e0198456.

41 Grams ME, Coresh J. Predicting risk of RRT in patients with CKD. Clin J Am Soc Nephrol 2017;12:3-4.

42 Venuthurupalli SK, Hoy WE, Healy HG, et al. CKD.QLD: establishment of a chronic kidney disease [CKD] registry in Queensland, Australia. BMC Nephrol 2017;18:1-10.

43 Kidney Health Australia. Chronic kidney disease (CKD) management in primary care. available online:, 2020. Available: https://kidney. org.au/uploads/resources/CKD-Management-in-Primary-Care handbook_2020.1.pdf [Accessed 09 Oct 2021].

44 Johnson DW. When to refer for specialist renal care. Available: https://www.cariguidelines.org/guidelines/chronic-kidney-disease/ early-chronic-kidney-disease/when-to-refer-for-specialist-renal-care/ [Accessed 10 Oct 2021].

45 Levin A, Stevens PE. Summary of KDIGO 2012 CKD guideline: behind the scenes, need for guidance, and a framework for moving forward. Kidney Int 2014;85:49-61.

46 Sumida K, Nadkarni GN, Grams ME, et al. Conversion of Urine Protein-Creatinine Ratio or Urine Dipstick Protein to Urine AlbuminCreatinine Ratio for Use in Chronic Kidney Disease Screening and Prognosis : An Individual Participant-Based Meta-analysis. Ann Intern Med 2020;173:426-35.

47 Tangri N, Ferguson T, Komenda P. Pro: risk scores for chronic kidney disease progression are robust, powerful and ready fo implementation. Nephrol Dial Transplant 2017;32:748-51.

48 Wojciechowski P, Tangri N, Rigatto C, et al. Risk prediction in CKD: the rational alignment of health care resources in CKD 4/5 care. Adv Chronic Kidney Dis 2016;23:227-30.

49 Reichel H, Zee J, Tu C, et al. Chronic kidney disease progression and mortality risk profiles in Germany: results from the chronic kidney disease outcomes and practice patterns study. Nephrol Dial Transplant 2020;35:803-10.

50 Evans M, Grams ME, Sang Y, et al. Risk factors for prognosis in patients with severely decreased GFR. Kidney Int Rep 2018;3:625-37.

51 Hoaglin DC, Iglewicz B. Fine-Tuning some resistant rules for outlier labeling. J Am Stat Assoc 1987;82:1147-9. 